\title{
Variational theory of the Ricci curvature tensor dynamics
}

\author{
Claudio Cremaschini ${ }^{1, a}$, Jiří Kovár ${ }^{1}$, Zdeněk Stuchlík $^{1}$, Massimo Tessarotto ${ }^{1,2}$ \\ ${ }^{1}$ Research Center for Theoretical Physics and Astrophysics, Institute of Physics, Silesian University in Opava, Bezručovo nám.13, \\ 74601 Opava, Czech Republic \\ ${ }^{2}$ Department of Mathematics and Geosciences, University of Trieste, Via Valerio 12, 34127 Trieste, Italy
}

Received: 6 November 2021 / Accepted: 16 November 2021 / Published online: 25 November 2021

(C) The Author(s) 2021

\begin{abstract}
In this letter a new Lagrangian variational principle is proved to hold for the Einstein field equations, in which the independent variational tensor field is identified with the Ricci curvature tensor $R^{\mu v}$ rather than the metric tensor $g_{\mu \nu}$. The corresponding Lagrangian function, denoted as $L_{R}$, is realized by a polynomial expression of the Ricci 4-scalar $R \equiv g_{\mu \nu} R^{\mu \nu}$ and of the quadratic curvature 4scalar $\rho \equiv R^{\mu \nu} R_{\mu \nu}$. The Lagrangian variational principle applies both to vacuum and non-vacuum cases and for its validity it demands a non-vanishing, and actually also positive, cosmological constant $\Lambda>0$. Then, by implementing the deDonder-Weyl formalism, the physical conditions for the existence of a manifestly-covariant Hamiltonian structure associated with such a Lagrangian formulation are investigated. As a consequence, it is proved that the Ricci tensor can obey a Hamiltonian dynamics which is consistent with the solutions predicted by the Einstein field equations.

A requisite that characterizes the mathematical formulation of physical laws concerns the possibility of deriving the fundamental dynamical equations of physical systems from least-action variational principles. This criterion applies to classical, quantum and relativistic mechanics as well as continuum field theories, where the existence of variational formulations is regarded "per se" as a property of consistency and correctness of the same physical laws. In fact, the Lagrangian and Hamiltonian formalisms established for their representations are crucial to understand the physical properties of classical and quantum fields, including for example their degrees of freedom and gauge properties, the role of constraints, the unveiling of symmetries and conservation laws.
\end{abstract}

The letter deals with the variational theory of the gravitational field in the framework of classical General Relativity (GR). The target addressed is twofold. First, it is proved that a manifestly-covariant variational Lagrangian theory can be constructed for the Ricci curvature tensor $R^{\mu \nu}$, to be treated

a e-mail: claudiocremaschini@gmail.com (corresponding author) as an independent variational tensor field but still yielding the Einstein field equations (EFE) as extremal equations. The present theory therefore does not produce $f(R)$-type modifications of standard fundamental GR equations, although - as we intend to show - it suggests also a way to produce them. These are generated: (a) by the inclusion of additional variational terms in the Lagrangian function depending on the Ricci tensor (e.g., Ref. [1]) or in the framework of socalled $f(R)$ gravity theories (e.g., Refs. [2,3]); (b) by the adoption of a new Hamiltonian action principle for the same fundamental equations of GR. Second, it is shown that the same Lagrangian theory admits a corresponding manifestlycovariant Hamiltonian theory of deDonder-Weyl type [4] which, under suitable conditions, is equivalent to the GR equations. This permits unveiling the existence of a Hamiltonian structure for EFE associated with the Ricci tensor dynamics and a symmetry existing between the variational treatments of the metric and Ricci tensors.

In order to introduce the subject, it is useful to briefly recall the main variational approaches to EFE available in the literature. We start with the original variational theory formulated by Hilbert, soon after confirmed by Einstein himself [5]. This is based on the adoption of the so-called Hilbert-Einstein (HE) asynchronous action functional

$S_{H E}(g(r)) \equiv \int_{\mathbf{M}^{4}} d \Omega L_{H E}(g)$,

where $S_{H E}(g(r))$ is considered a functional dependent only on the variational metric tensor $g(r) \equiv\left\{g_{\mu \nu}\right\}$. In HE theory, the latter belongs by assumption to a suitably-constrained functional setting $\{g(r)\}_{C}$, referred to as asynchronous functional setting [6]. In the same setting, the invariant 4-volume element of the Riemann space-time $\left\{\mathbf{M}^{4}, g(r)\right\}$, namely $d \Omega \equiv d^{4} r \delta \sqrt{-|g(r)|}$ (with $d^{4} r \equiv \prod_{i=0,3} d r^{i}$ being the canonical measure of the space-time manifold $\mathbf{M}^{4}$ ), depends explicitly on the determinant of $g(r)$, i.e., $|g(r)|$. In addition, here $L_{H E}(g)$ denotes a suitable Lagrangian 4-scalar 
function, while the quantity $\sqrt{-|g|} L_{H E}(g)$ identifies the corresponding variational Lagrangian. Hence, the variational Lagrangian characterizing HE theory is not a 4-scalar, but identifies a so-called 4-scalar density. In the same framework the generalized Lagrangian coordinates can be identified (in any GR-frame) with the independent 4-tensor components of the symmetric variational metric tensor itself, i.e., $g(r) \equiv\left\{g_{\mu \nu}\right\}$. At the same time, however, any 4-tensor $g(r) \in\{g(r)\}_{C}$ realizes also a metric tensor, so that its countervariant and covariant components respectively raise and lower tensor indices and thus necessarily must satisfy by construction the orthogonality condition $g_{\mu \nu} g^{\mu k}=\delta_{\nu}^{k}$. The latter implies in turn the "normalization" condition $g_{\mu \nu}(r) g^{\mu \nu}(r)=4$. As a further consequence, in the same functional setting, $g(r)$ must determine also the Christoffel symbols $\Gamma(g(r))$ and the Ricci tensor $R_{\mu \nu}(g)$. Consequently this means that $g(r)$ must satisfy the well-known metric compatibility condition, so that its covariant derivatives vanish identically. The HE 4-scalar Lagrangian $L_{H E}(g)$ is defined as

$L_{H E}(g)=V_{H E}(g)+V_{F}$,

where

$V_{H E}(g)=-\frac{c^{3}}{16 \pi G}\left(g^{\mu v} R_{\mu \nu}(g)-2 \Lambda\right)$

denotes the gravitational contribution, with $\Lambda>0$ being the cosmological constant and where $G$ is the Newton constant of gravitation and $c$ is the speed of light in vacuum. Instead, $V_{F} \equiv V_{F}(g, r)$ is the non-vacuum contribution due to possible external fields to be prescribed in terms of the field Lagrangian $L_{F}$ as $V_{F}=\frac{1}{c} L_{F}$. The $\mathrm{HE}$ variational principle is then obtained by requiring that for arbitrary variations $\delta g(r)$ it must be

$$
\begin{aligned}
& \left.\delta S_{H E}(g(r))\right|_{g=\widehat{g}(r)} \\
& \quad=\left.\frac{d}{d \theta} S_{H E}(\widehat{g}(r)+\theta \delta g(r))\right|_{\theta=0}=0,
\end{aligned}
$$

with the symbol $\delta$ denoting the Frechet derivative and $\widehat{g}(r)$ being the extremal classical metric tensor, to be ultimately identified with the solution of EFE. A characteristic feature of the HE variational theory is that $d \Omega$ yields nonvanishing variational contributions to $\delta S_{H E}(g(r))$, since $\delta d \Omega=d^{4} r \delta \sqrt{-|g|}$, where $\delta \sqrt{-|g|}=-\frac{1}{2} \sqrt{-|g|} g_{\mu \nu} \delta g^{\mu \nu}$. As a consequence, the variation of the functional $S_{H E}(g(r))$ does not preserve the space-time volume element, and for this reason is referred to as asynchronous [7]. Straightforward algebra [6] then delivers

$$
\left.\delta S_{H E}(g(r))\right|_{g=\widehat{g}(r)}=\left.\delta S_{H E}(g)\right|_{\operatorname{expl}}+\left.\delta S_{H E}(g)\right|_{\text {impl }},
$$

where the implicit contribution is

$$
\left.\delta S_{H E}(g)\right|_{\mathrm{impl}}=\int_{\mathbf{M}^{4}} d \Omega\left[-\frac{c^{3}}{16 \pi G} \widehat{g}^{\alpha \beta} \frac{\delta R_{\alpha \beta}}{\delta g^{\mu \nu}}\right] \delta g^{\mu \nu},
$$

while the explicit contributions can be written as

$$
\left.\delta S_{H E}(g)\right|_{\text {expl }}=\int_{\mathbf{M}^{4}} d^{4} r\left[A_{\mu \nu}+B_{\mu \nu}+C_{\mu \nu}\right] \delta g^{\mu \nu},
$$

where $A_{\mu \nu}, B_{\mu \nu}$ and $C_{\mu \nu}$ are tensor densities, respectively defined as

$$
\begin{aligned}
A_{\mu \nu} & \equiv L_{H E} \frac{\delta \sqrt{-|g|}}{\delta g^{\mu \nu}}, \\
B_{\mu \nu} & \equiv-\frac{c^{3}}{16 \pi G} \sqrt{-|g|} R_{\alpha \beta} \frac{\delta g^{\alpha \beta}}{\delta g^{\mu \nu}}, \\
C_{\mu \nu} & \equiv \frac{1}{c} \sqrt{-|g|} \frac{\delta L_{F}}{\delta g^{\mu \nu}} .
\end{aligned}
$$

As shown in Ref. [6], in order to recover the correct form of EFE, the constraint condition $\left(\delta S_{H E}(g)\right)_{\text {impl }}=0$ must hold. This requires in turn an appropriate treatment of the implicit contributions carried by the Ricci tensor, which depends on the second-order partial derivatives of the metric tensor. Then, the requirement that $\left.\delta S_{H E}(g)\right|_{\text {expl }}=0$ holds for arbitrary variations $\delta g^{\mu \nu}(r)$ yields the Einstein equations

$\widehat{R}_{\mu \nu}-\left(\frac{1}{2} \widehat{R}-\Lambda\right) \widehat{g}_{\mu \nu}=\kappa \widehat{T}_{\mu \nu}$,

where $\widehat{R}_{\mu \nu}=R_{\mu \nu}(\widehat{g}(r))$ and $\widehat{R}=\widehat{g}^{\mu \nu}(r) \widehat{R}_{\mu \nu} \equiv R(\widehat{g}(r))$ denote respectively the background Ricci 4-tensor and Ricci 4-scalar, $\widehat{T}_{\mu \nu}=T_{\mu \nu}(\widehat{g}(r))$ is the background stress-energy tensor associated with the external source fields described by the external-field Lagrangian density $L_{F}(g)$ and $\kappa$ denotes the universal constant $\kappa=8 \pi G / c^{4}$.

The asynchronous HE approach is characterized by a number of conceptual deficiencies, which pertain primarily the validity of the principle of manifest covariance and of fundamental gauge invariance properties to be satisfied by the HE Lagrangian function [6,7]. An alternative approach which overcomes these problems is provided by the synchronous variational principle, which realizes for the gravitational field a deDonder-Weyl manifestly-covariant variational theory $[8,9]$. The synchronous variational approach is characterized by the use of a 4-scalar Lagrangian function expressed in terms of superabundant variables $g_{\mu \nu}$ and $\widehat{g}_{\mu \nu}$. In this setting, the variational tensor $g \equiv\left\{g_{\mu \nu}\right\}$ is distinguished from a suitably-defined "prescribed" (i.e., non-variational) background metric tensor $\widehat{g} \equiv\left\{\widehat{g}_{\mu \nu}\right\}$, which defines uniquely the covariance properties of the theory and, as such, is ultimately assumed to be determined by the extremal EulerLagrange equations (i.e., EFE). Hence, $\widehat{g}$ expresses the geometric character of the metric tensor, namely it satisfies the orthogonality condition $\widehat{g}_{\mu \nu} \widehat{g}^{\mu k}=\delta_{\nu}^{k}$, so that it raises/lowers tensor indices, as well the metric compatibility condition $\widehat{\nabla}_{\alpha} \widehat{g}_{\mu \nu}=0$, so that it defines the standard Christoffel connections and curvature tensors of space-time. On the contrary, in this framework the variational tensor $g$ is such that $g_{\mu \nu} g^{\mu k} \neq \delta_{\nu}^{k}$. The distinction between $g$ and $\widehat{g}$ holds only at 
the variational level, since in the (extremal) EFE the identity $g=\widehat{g}$ is restored. In the synchronous setting, hatted quantities depend on the background metric tensor $\widehat{g}$ and do not contribute to the variational calculus. Thus, denoting in particular the synchronous volume element as $d \widehat{\Omega}=d^{4} r \sqrt{-|\widehat{g}|}$, its variation vanishes by construction so that $\delta d \widehat{\Omega}=0$. This volume-preserving property under the action of the operator $\delta$ justifies the name given to this approach as the synchronous variational principle, in contrast to the asynchronous theory.

The corresponding synchronous Lagrangian action functional is thus defined as

$S_{s}(g(r), \widehat{g}(r))=\int_{\mathbf{M}^{4}} d \widehat{\Omega} L_{S}(g, \widehat{g})$,

where $S_{S}(g(r), \widehat{g}(r))$ is considered a functional dependent only on the variational tensor (not a metric tensor) $g(r) \equiv\left\{g_{\mu \nu}\right\}$, with $\widehat{g}(r)$ being instead identified with a prescribed background metric field tensor, i.e., the solution of EFE. Here, $L_{s}(g, \widehat{g}) \equiv L_{s}(g(r), \widehat{g}(r))$ is the variational Lagrangian and, in contrast to the asynchronous action functional (1), the volume element takes the form $d \widehat{\Omega}$. The variational Lagrangian can be prescribed so that

$L_{s}(g, \widehat{g}) \equiv h(g, \widehat{g}) L(g, \widehat{g})$,

where

$h(g, \widehat{g})=2-\frac{1}{4} g^{\eta \beta}(r) g^{\mu \nu}(r) \widehat{g}_{\eta \mu}(r) \widehat{g}_{\beta \nu}(r)$

identifies a 4-scalar, i.e., the variational weight-factor, which is defined so that $h(\widehat{g}, \widehat{g})=1$. Instead, the 4-scalar Lagrangian $L(g, \widehat{g})$ can be identified with a form analogous to the HE 4-scalar Lagrangian $L_{H E}(g)$ given above by Eq. (2), namely of the form

$L(g, \widehat{g})=V_{G}(g, \widehat{g})+V_{F}(g, \widehat{g})$,

where now

$$
\begin{aligned}
V_{G}(g, \widehat{g}) & \equiv-\frac{c^{3}}{16 \pi G}\left(g^{\mu \nu} \widehat{R}_{\mu \nu}-2 \Lambda\right), \\
\widehat{R}_{\mu \nu} & \equiv R_{\mu \nu}(\widehat{g}),
\end{aligned}
$$

and $V_{F}(g, \widehat{g})=\frac{1}{c} L_{F}(g, \widehat{g})$. Then, the synchronous Lagrangian action principle follows by prescribing

$$
\left.\delta S_{S}(g(r), \widehat{g}(r))\right|_{g=\widehat{g}}=0,
$$

for arbitrary variations $\delta g(r)$, while noting that $\delta \widehat{g}(r) \equiv 0$. Here, the symbol $\delta$ denotes the variation operator, i.e., the Frechet derivative

$$
\left.\left.\delta S_{S}(g(r), \widehat{g}(r))\right|_{g=\widehat{g}} \equiv \frac{d}{d \theta} S_{L}(\widehat{g}(r)+\theta \delta g(r), \widehat{g}(r))\right|_{\theta=0}(1.9)
$$

By noting that $\delta h(g, \widehat{g})=-\frac{1}{2} \widehat{g}^{\mu \nu}(r) \delta g_{\mu \nu}$, the evaluation of $\left.\delta S_{s}(g(r), \widehat{g}(r))\right|_{g=\widehat{g}(r)}$ is straightforward. In fact, in the synchronous setting only explicit dependences on $g$ give a contribution, while the implicit ones carried by the Ricci tensor are now excluded. Hence, from Eq. (18) one recovers EFE in the correct form (11).

In the synchronous setting, $g_{\mu \nu}$ is not a metric tensor, so that $\widehat{\nabla}_{\alpha} g_{\mu \nu} \neq 0$. Hence, it is possible to determine a more general form of the synchronous Lagrangian function which includes also terms proportional to the covariant derivatives $\widehat{\nabla}_{\alpha} g_{\mu \nu}$, to be interpreted as "generalized field velocities". Under the requisite of preserving the correct form of EFE, by implementing a deDonder-Weyl approach this leads to the formulation of a classical manifestly-covariant Hamiltonian theory of GR (see Refs. [8-10]). We notice that a generalization of this type can only be achieved for the synchronous variational principle, while this possibility remains forbidden for the asynchronous formulation in which the derivatives of the corresponding variational metric tensor $g(r)$ are identically vanishing. More precisely, the target is met by introducing the effective kinetic energy $T$ defined as

$T=-\frac{c^{3}}{16 \pi G}\left(\frac{1}{2} \widehat{\nabla}^{\eta} g_{\mu \nu} \widehat{\nabla}_{\eta} g^{\mu \nu}\right)$,

which has the same dimensions of $L_{S}$, and the corresponding 4-scalar Lagrangian function $L_{T}(g, \widehat{\nabla} g, \widehat{g})=$ $L_{T}(g(r), \widehat{\nabla} g(r), \widehat{g}(r))$ as

$L_{T}(g, \widehat{\nabla} g, \widehat{g})=T+L_{s}(g, \widehat{g})$.

Then, upon identifying the 4-tensor conjugate canonical momentum of $g_{\mu \nu}$ with

$\Pi_{\mu \nu}^{\eta}(r)=\frac{\partial L_{T}(g, \widehat{\nabla} g, \widehat{g})}{\partial \widehat{\nabla}_{\eta} g^{\mu \nu}}$,

the change of Lagrangian variables into canonical variables can be made. The Legendre transform then relates the Lagrangian and the Hamiltonian function as

$L_{T}(g, \Pi, \widehat{g}) \equiv \Pi_{\mu \nu}^{\eta} \widehat{\nabla}_{\eta} g^{\mu \nu}-H_{T}(g, \Pi, \widehat{g})$,

where, omitting for simplicity dimensional constants, $H_{T}(g, \Pi, \widehat{g})$ is the 4-scalar variational Hamiltonian defined by

$H_{T}(g, \Pi, \widehat{g})=\frac{1}{2} \Pi_{\mu \nu}^{\eta}(r) \Pi_{\eta}^{\mu \nu}(r)+L_{s}(g, \widehat{g})$.

Then, by introducing the Hamilton functional

$S_{H}(g(r), \Pi(r), \widehat{g}(r))=\int_{\mathbf{M}^{4}} d \widehat{\Omega} L_{T}(g, \Pi, \widehat{g})$

and the Hamiltonian variational principle (in terms again of the Frechet derivative)

$\left.\delta S_{H}(g(r), \Pi(r), \widehat{g}(r))\right|_{g=\widehat{g}(r)}=0$,

a set of Hamilton equations is obtained

$\left\{\begin{array}{c}\widehat{\nabla}_{\eta} g^{\mu \nu}=\frac{\partial H_{T}(g, \Pi, \widehat{g})}{\partial \Pi_{\mu \nu}^{\eta}} \\ \widehat{\nabla}_{\eta} \Pi_{\mu \nu}^{\eta}=-\frac{\partial H_{T}(g, \Pi, \widehat{g})}{\partial g^{\mu \nu}}\end{array}\right.$. 
These equations are equivalent to the Lagrangian equations and include the additional contribution due to nonvanishing canonical momenta. Upon evaluating them for $(g(r), \Pi(r))=(\widehat{g}(r), \widehat{\Pi}(r) \equiv 0)$ one recovers EFE (the detailed proof can be found in Ref. [8]). The result shows that in the framework of the synchronous variational principle, EFE admit a classical Hamiltonian structure which obeys manifestly-covariant Lagrangian and Hamiltonian equations.

Both cases discussed above display the peculiarity of the variational calculation of $R_{\mu \nu}$ with respect to the tensor $g_{\mu \nu}$, suggesting the conjecture that a deeper inspection of its meaning might disclose the existence of novel theoretical features concerning the variational formulation of EFE. Starting from these considerations, the purpose in the following is to prove the existence of an alternative route, which preserves the consistency with the principle of manifest covariance (as the synchronous approach) but is built on the identification of $R_{\mu \nu}$ as the independent variational tensor for the variational theory of EFE, rather than $g_{\mu \nu}$. This approach is shown to be consistent with both Lagrangian and deDonder-Weyl Hamiltonian variational formulations of GR equations. This in turn yields a novel point of view for the theoretical interpretation of the physical meaning of EFE and the underlying Hamiltonian structure.

The proof of the statement is based on the introduction of a Ricci-functional of the form

$S_{R}(\widehat{g}(r), R(r)) \equiv \int_{\mathbf{M}^{4}} d \widehat{\Omega} L_{R}(\widehat{g}, R)$,

which is considered dependent on the variational tensor field $R(r) \equiv\left\{R_{\mu \nu}(r)\right\}$, with $R(r)$ belonging to a suitable synchronous variational setting. This means, more precisely that, as in all synchronous variational principles, the 4-tensor field $\widehat{g}(r)$ identifies the prescribed metric field tensor solution of EFE. Thus, in particular, the 4-scalar variational RicciLagrangian function $L_{R}(\widehat{g}, R)$ is taken here of the form

$$
\begin{aligned}
L_{R}(\widehat{g}, R) \equiv & -\frac{c^{3}}{16 \pi G}\left(\frac{1}{2 \Lambda} \rho+R-\frac{1}{4 \Lambda} R^{2}\right) \\
& +\frac{1}{2 c} \frac{1}{\Lambda} R^{\mu \nu} \widehat{T}_{\mu \nu},
\end{aligned}
$$

with $\rho \equiv R^{\mu \nu} R_{\mu \nu}$, and $R \equiv R^{\mu \nu} \widehat{g}_{\mu \nu}$ denoting the corresponding variational Ricci 4-scalar. A number of remarks must be pointed out concerning the mathematical properties of $L_{R}(\widehat{g}, R)$ :

1. The first one is that in $S_{R}$ the two tensor fields $R^{\mu \nu}$ and $\widehat{g}_{\mu \nu}$ are independent. The variational tensor is identified with $R^{\mu \nu}$, so that the hatted tensor $\widehat{g}_{\mu \nu}$ is effectively considered as extremal in such a framework, and therefore it is a metric tensor. Accordingly, $R^{\mu \nu} R_{\mu \nu} \equiv$ $R^{\mu \nu} R^{\alpha \beta} \widehat{g}_{\alpha \mu} \widehat{g}_{\beta \nu}$, while the stress-energy tensor $\widehat{T}_{\mu \nu}$ is regarded as a function of extremal fields in such a representation.

2. The dimensional units have been included so to make the Lagrangian $L_{R}(\widehat{g}, R)$ homogeneous with $L_{H E}$ and $L_{s}$, so that $L_{R}(\widehat{g}, R)$ is an action. Accordingly, the stressenergy tensor $\widehat{T}_{\mu \nu}$ has the same dimension of the externalfield Lagrangian $L_{F}$ introduced above.

3. The Lagrangian $L_{R}(\widehat{g}, R)$ is a polynomial function that contains a linear and a quadratic contributions in the Ricci 4-scalar $R$, a quadratic term in the Ricci tensor $R^{\mu \nu}$ which enters through the curvature 4-scalar $\rho \equiv R^{\mu \nu} R_{\mu \nu}$ and a linear term in the Ricci tensor $R^{\mu \nu}$ that carries the coupling with external sources.

However, from the conceptual point of view the most striking feature that emerges from Eq. (29) is that the Lagrangian $L_{R}(\widehat{g}, R)$ depends on the cosmological constant $\Lambda$. Its inclusion is necessary in order for $L_{R}(\widehat{g}, R)$ to have the correct dimension of an action, since $[\Lambda]=L^{-2}$, and at the same time to warrant recovering the correct form of EFE as the extremal equations derived from it (see below). In addition, we notice that necessarily the constraint $\Lambda \neq 0$ is demanded (which permits, in turn, the choice $\Lambda>0$ ). These features therefore suggest the interesting role taken by the cosmological constant in this approach to the derivation of the classical EFE based on the variational theory for the Ricci tensor, implying that $\Lambda$ must be a foundational element of GR theory. In fact, the role of $\Lambda>0$ can be of high relevance in astrophysics, as summarized in Ref. [11]. Remarkably, the choice of a positive cosmological constant in the variational principle for EFE is a condition in agreement with the result pointed out in Ref. [12] where it was shown that $\Lambda>0$ can be associated with the validity of the orthogonality condition $\widehat{g}_{\mu \nu} \widehat{g}^{\mu k}=\delta_{\nu}^{k}$ for the extremal metric tensor. Finally, the crucial role of $\Lambda$ pointed out here is also consistent with its meaning which arises in the manifestly-covariant quantum gravity theory and the relationship between quantum-modified and classical GR equations [12,13].

The Ricci-Lagrangian variational principle associated with the action integral $S_{R}(\widehat{g}(r), R(r))$ can then be obtained by requiring that for arbitrary variations $\delta R(r) \equiv \delta R^{\mu \nu}(r)$ it occurs, for arbitrary variations $\delta R(r)$, that

$$
\begin{aligned}
& \left.\delta S_{R}(\widehat{g}(r), R(r))\right|_{R=\widehat{R}(r)} \\
& =\left.\frac{d}{d \theta} S_{R}(\widehat{R}(r)+\theta \delta R(r))\right|_{\theta=0}=0,
\end{aligned}
$$

with the symbol $\delta$ denoting the Frechet derivative and the variation being performed with respect to the independent tensor field $R^{\mu \nu}(r)$. Notice that here the extremal field $\widehat{R}(r)$, in difference with respect to Eq. (17), may be considered for greater generality independent of the background metric tensor field $\widehat{g}(r)$. Equation (30) is thus equivalent to the tensorial equation 
$\frac{\delta L_{R}(\widehat{g}, R)}{\delta R^{\mu \nu}(r)}=0$,

with $L_{R}(\widehat{g}, R)$ being given by Eq. (29). It is then immediate to verify that the explicit algebraic calculation of the previous equation yields the correct form of EFE, namely Eq. (11). The following remarkable interpretation follows: the realization of EFE can be reached by extremizing two classes of Lagrangian functions, respectively $L_{H E}$ or $L_{S}$ in terms of the tensor field $g_{\mu \nu}(r)$ and $L_{R}$ for the curvature Ricci tensor $R^{\mu \nu}(r)$. In addition, we stress that for the Ricci-Lagrangian variational action principle the distinction between synchronous/asynchronous approaches is irrelevant. This is because in both cases all variations of the metric tensor do not occur. So that the validity of the new action principle appears of general validity in this respect.

We notice that the Lagrangian variational principle (30) can apply both to vacuum and non-vacuum cases. The contribution in $L_{R}(\widehat{g}, R)$ describing the coupling term between the curvature and external source fields is the 4-scalar $\frac{1}{\Lambda} R^{\mu \nu} \widehat{T}_{\mu \nu}$. This kind of coupling is different from that occurring in the action principles $S_{H E}(g(r))$ and $S_{S}(g(r), \widehat{g}(r))$, where the relationship is between the variational tensor $g_{\mu \nu}(r)$ and the Lagrangian $L_{F}$ of source fields. In the case of $S_{R}(\widehat{g}(r), R(r))$ instead the coupling occurs directly between the stress-energy tensor $\widehat{T}_{\mu \nu}$ and the Ricci variational tensor $R^{\mu \nu}$. Remarkably, however, this form of interaction is not unconventional. In fact, this term exhibits a structure analogous to that occurring in classical electrodynamics between the electromagnetic 4-potential $A_{\mu}$ and the 4-current $J^{\mu}$, namely proportional to the 4-scalar $A_{\mu} J^{\mu}$, where $A_{\mu}$ is the variational field and $J^{\mu}$ is considered as prescribed [14]. We point out that this type of coupling could be an advantage for the variational description of continuum fields in those cases in which one knows the form of the stress-energy tensor (e.g., through symmetry or conservation-law criteria) but not the details of the underlying Lagrangian description. An example of this type is represented by continuum fluids, possibly in the presence of non-ideal phenomena like viscous or dissipation effects, torsion, diffusion, boundary-layer interactions.

We now pose the question of establishing whether the Lagrangian solution $L_{R}(\widehat{g}, R)$ admits the existence of a corresponding Hamiltonian theory, again to be established in terms of a manifestly-covariant deDonder-Weyl approach, and if this yields Hamiltonian equations which are equivalent to EFE. The program is analogous to that carried out for the tensor $g_{\mu \nu}$ in the synchronous variational principle. In order to proceed, for the convenience of calculations, we first write the Lagrangian (29) in the compact form

$L_{R}(\widehat{g}, R) \equiv-\left(\frac{1}{2 \Lambda} \rho+R-\frac{1}{4 \Lambda} R^{2}\right)+\frac{\kappa}{2 \Lambda} R^{\mu \nu} \widehat{T}_{\mu \nu}$ where all universal constants are included into the symbol $\kappa$. Then, we introduce the "generalized velocity field" associated with the covariant derivative of $R^{\mu \nu}$, namely the quantity $\widehat{\nabla}_{\eta} R^{\mu \nu}$. For the variational Ricci tensor $R^{\mu \nu}$ one generally has that $\widehat{\nabla}_{\eta} R^{\mu \nu} \neq 0$. Therefore, we can define the effective Ricci-kinetic energy $T_{R} \equiv T_{R}(\widehat{g}, \widehat{\nabla} R)$ as

$T_{R}=-\frac{1}{2 \Lambda^{2}} \widehat{\nabla}^{\eta} R_{\mu \nu} \widehat{\nabla}_{\eta} R^{\mu \nu}$,

which has the same dimensions of $L_{R}(\widehat{g}, R)$. The corresponding 4-scalar Lagrangian function $L_{T_{R}}(\widehat{g}, R, \widehat{\nabla} R)=$ $L_{T_{R}}(\widehat{g}(r), R(r), \widehat{\nabla} R(r))$ which includes this contribution becomes

$L_{T_{R}}(\widehat{g}, R, \widehat{\nabla} R)=T_{R}(\widehat{g}, \widehat{\nabla} R)+L_{R}(\widehat{g}, R)$.

Then, the 4-tensor conjugate canonical momenta of $R_{\mu \nu}$ are defined accordingly as

$\pi_{\mu \nu}^{\eta}(r)=\frac{\partial L_{T_{R}}(\widehat{g}, R, \widehat{\nabla} R)}{\partial \widehat{\nabla}_{\eta} R^{\mu \nu}}$,

which have one tensorial index more than the conjugate coordinate field (i.e., $R^{\mu v}$ ), as it is characteristic of the deDonderWeyl solution. One obtains explicitly

$\pi_{\mu \nu}^{\eta}(r)=\frac{1}{\Lambda^{2}} \widehat{\nabla}^{\eta} R_{\mu \nu}$.

With this definition we can now proceed introducing the Ricci-Hamiltonian function $H_{T_{R}}(\widehat{g}, R, \pi)$ related to the Lagrangian function by means of the customary Legendre transform as

$L_{T_{R}}(\widehat{g}, R, \pi) \equiv \pi_{\mu \nu}^{\eta} \widehat{\nabla}_{\eta} R^{\mu \nu}-H_{T_{R}}(\widehat{g}, R, \pi)$,

where $H_{T_{R}}(\widehat{g}, R, \pi)$ is the 4-scalar variational Hamiltonian function defined by

$H_{T_{R}}(\widehat{g}, R, \pi)=\frac{1}{2} \Lambda^{2} \pi_{\mu \nu}^{\eta}(r) \pi_{\eta}^{\mu \nu}(r)+L_{T_{R}}(\widehat{g}, R, \pi)$.

The Hamiltonian structure is therefore defined by the set $\left\{\mathbf{M}^{4}, x_{R}\right\}$, where the Ricci canonical state $x_{R}$ is formed by the couple of conjugate variables $x_{R} \equiv\left(R^{\mu \nu}, \pi_{\mu \nu}^{\eta}\right)$. In terms of this expression we can introduce the Ricci-Hamilton functional

$S_{H_{R}}(\widehat{g}(r), R(r), \pi(r))=\int_{\mathbf{M}^{4}} d \widehat{\Omega} L_{T_{R}}(\widehat{g}, R, \pi)$

and the Hamilton variational principle

$\delta S_{H_{R}}(\widehat{g}(r), R(r), \pi(r)) \mid=0$,

where $\delta$ denotes again the Frechet derivative. As a consequence, this provides the equivalent set of Hamilton equations in tensorial form

$\left\{\begin{array}{c}\widehat{\nabla}_{\eta} R^{\mu \nu}=\frac{\partial H_{T_{R}}(\widehat{g}, R, \pi)}{\partial \pi_{\mu \nu}^{\eta}} \\ \widehat{\nabla}_{\eta} \pi_{\mu \nu}^{\eta}=-\frac{\partial H_{T_{R}}(\widehat{g}, R, \pi)}{\partial R^{\mu \nu}}\end{array}\right.$. 
An explicit calculation then yields

$$
\left\{\begin{array}{c}
\widehat{\nabla}_{\eta} R^{\mu \nu}=\Lambda^{2} \pi_{\eta}^{\mu \nu}(r) \\
\widehat{\nabla}_{\eta} \pi_{\mu \nu}^{\eta}=-\frac{\partial L_{T_{R}}(\widehat{g}, R, \pi)}{\partial R^{\mu \nu}}
\end{array},\right.
$$

where the first equation recovers as usual the definition of the canonical momentum in terms of the generalized velocity, i.e., Eq. (36). The second equation instead is a generalization of EFE with the additional contribution associated with the canonical momentum $\pi_{\mu \nu}^{\eta}$. In fact, evaluating it for the extremal fields and using the first equation of (41) gives

$\frac{1}{\Lambda} \square \widehat{R}_{\mu \nu}+\widehat{R}_{\mu \nu}-\left(\frac{1}{2} \widehat{R}-\Lambda\right) \widehat{g}_{\mu \nu}=\kappa \widehat{T}_{\mu \nu}$,

where $\square$ is the D'Alembertian differential operator $\square \equiv$ $\widehat{\nabla}_{\eta} \widehat{\nabla}^{\eta}$.

Equation (42) can differ from EFE, since in general $\square \widehat{R}_{\mu \nu} \neq 0$. The equivalence is reached necessarily iff $\square \widehat{R}_{\mu \nu}=0$, which is realized for example when separately $\widehat{\nabla}_{\eta} \widehat{R}_{\mu \nu}=0$. Incidentally, the same type of mathematical condition arises also in the manifestly-covariant Hamiltonian theory of GR based on the synchronous action integral (25) (see Eq. (27) and subsequent discussion). This condition on $\widehat{R}_{\mu \nu}$ is satisfied trivially in vacuum when $\widehat{T}_{\mu \nu}=0$, since in such a case the general solution of EFE is the Einstein space $\widehat{R}_{\mu \nu}=\Lambda \widehat{g}_{\mu \nu}$ [15], for which $\widehat{\nabla}_{\eta} \widehat{R}_{\mu \nu}=\Lambda \widehat{\nabla}_{\eta} \widehat{g}_{\mu \nu}=0$ thanks to the metric compatibility condition holding for the metric tensor. More generally, a wider class of admissible Hamiltonian solutions is obtained also in non-vacuum cases for a suitable choice of stress-energy tensor which must be of the functional type $\widehat{T}_{\mu \nu}=\widehat{T}_{\mu \nu}\left(\widehat{g}_{\mu \nu}\right)$. The latter condition includes for example the screening effect of the cosmological constant due to quantum-gravity corrections to EFE predicted in the framework of the manifestly-covariant quantum gravity theory (see Ref. [16]). Hence, even though the validity of the Ricci Hamiltonian theory is subject to precise restrictions, these are general enough to be effectively realized concretely in physical systems of cosmological and astrophysical interests where the Ricci tensor obeys a Hamiltonian dynamics which is consistent with the tensorial form of the space-time solutions predicted by EFE.

In summary, in this letter we have proved the validity of a novel variational principle for the Einstein field equations in which the independent variational tensor field is identified with the Ricci curvature tensor $R^{\mu \nu}$ rather than the metric tensor $g_{\mu \nu}$. The corresponding Lagrangian function, denoted here as $L_{R}$, is shown to depend both on the Ricci 4-scalar $R \equiv g_{\mu \nu} R^{\mu \nu}$ through a polynomial of 2 nd degree and on the curvature 4-scalar $\rho \equiv R^{\mu \nu} R_{\mu \nu}$, together with the simultaneous necessary condition of existence of a positive cosmological constant $\Lambda>0$. Remarkably, the new Lagrangian variational principle can apply both to vacuum and non-vacuum cases. This result permits to conjecture the physical interpretation according to which the Einstein field equations can also be understood as (extremal) Lagrangian equations for the Ricci tensor dynamics that satisfy the leastaction principle expressed in terms of $L_{R}$. As a further result, starting from $L_{R}$ and implementing the deDonder-Weyl formalism, the possibility of obtaining a manifestly-covariant Hamiltonian structure associated with the novel Lagrangian formulation has been investigated. It is proved that this conclusion can be satisfied for a class of GR space-times including the vacuum case generating the Einstein space solution as well for suitable prescription of the functional form of the stress-energy tensor associated with external source fields.

Acknowledgements CC, JK and ZS acknowledge the support of the Research Center for Theoretical Physics and Astrophysics, Institute of Physics, Silesian University in Opava, Czech Republic. ZS acknowledges support of the grant 19-03950S of the Czech Science Foundation (GAČR).

Data Availability Statement This manuscript has no associated data or the data will not be deposited. [Authors' comment: This is a theoretical research. All data produced during this study are contained in this published letter.]

Open Access This article is licensed under a Creative Commons Attribution 4.0 International License, which permits use, sharing, adaptation, distribution and reproduction in any medium or format, as long as you give appropriate credit to the original author(s) and the source, provide a link to the Creative Commons licence, and indicate if changes were made. The images or other third party material in this article are included in the article's Creative Commons licence, unless indicated otherwise in a credit line to the material. If material is not included in the article's Creative Commons licence and your intended use is not permitted by statutory regulation or exceeds the permitted use, you will need to obtain permission directly from the copyright holder. To view a copy of this licence, visit http://creativecomm ons.org/licenses/by/4.0/.

Funded by $\mathrm{SCOAP}^{3}$.

\section{References}

1. B. Li, J.D. Barrow, D.F. Mota, Cosmology of Ricci-tensor-squared gravity in the Palatini variational approach. Phys. Rev. D 76, 104047 (2007)

2. T. Karakasis, E. Papantonopoulos, Z. Tang, B. Wang, Exact black hole solutions with a conformally coupled scalar field and dynamic Ricci curvature in $\mathrm{f}(\mathrm{R})$ gravity theories. Eur. Phys. J. C 81, 897 (2021)

3. S. Chakraborty, D. Gregoris, Cosmological evolution with quadratic gravity and nonideal fluids. Eur. Phys. J. C 81, 944 (2021)

4. J. Struckmeier, A. Redelbach, Covariant Hamiltonian field theory. Int. J. Mod. Phys. E 17, 435 (2008)

5. A. Einstein, The Meaning of Relativity (Princeton University Press, Princeton, 2004)

6. M. Tessarotto, C. Cremaschini, The principle of covariance and the Hamiltonian formulation of general relativity. Entropy 23, 215 (2021)

7. C. Cremaschini, M. Tessarotto, Synchronous Lagrangian variational principles in general relativity. Eur. Phys. J. Plus 130, 123 (2015) 
8. C. Cremaschini, M. Tessarotto, Hamiltonian approach to GRpart 1: covariant theory of classical gravity. Eur. Phys. J. C 77, 329 (2017)

9. C. Cremaschini, M. Tessarotto, Hamiltonian approach to GRpart 2: covariant theory of quantum gravity. Eur. Phys. J. C 77, 330 (2017)

10. C. Cremaschini, M. Tessarotto, Coupling of quantum gravitational field with Riemann and Ricci curvature tensors. Eur. Phys. J. C 81, 548 (2021)

11. Z. Stuchlík, M. Kološ, J. Kovář, P. Slaný, A. Tursunov, Influence of cosmic repulsion and magnetic fields on accretion disks rotating around Kerr black holes. Universe 6, 26 (2020)

12. C. Cremaschini, M. Tessarotto, Classical variational theory of the cosmological constant and its consistency with quantum prescription. Symmetry 12, 633 (2020)
13. C. Cremaschini, M. Tessarotto, Space-time second-quantization effects and the quantum origin of cosmological constant in covariant quantum gravity. Symmetry 10, 287 (2018)

14. L.D. Landau, E.M. Lifschitz, Field Theory, Theoretical Physics, vol. 2 (Addison-Wesley, New York, 1957)

15. A.Z. Petrov, Einstein Spaces (Pergamon, Oxford, 1969)

16. C. Cremaschini, M. Tessarotto, Quantum-gravity screening effect of the cosmological constant in the de Sitter space-time. Symmetry 12, $531(2020)$ 